\title{
The effects of illumination and trophic strategy on gene expression in Chlamydomonas reinhardtii
}

\author{
Victor Sanchez-Tarre, Alexandros Kiparissides \\ Department of Biochemical Engineering, University College London, WC1E 6BT, London, UK
}

\section{A R T I C L E I N F O}

\section{Keywords:}

Green microalgae

Wavelength selection

RT-qPCR

Carbon metabolism

Reference genes

\begin{abstract}
A B S T R A C T
Microalgae are of substantial biotechnological interest due their polyphyletic nature which grants them access to a wide array of high-value metabolites. The inherent genetic diversity of microalgae combined with their metabolic plasticity when grown using different trophic and illumination strategies necessitate the establishment of a reference knowledge base. In the present study we present a detailed characterisation of the combined effects of wavelength selection and trophic strategy on the growth kinetics and gene expression profile of the model microalgae Chlamydomonas reinhardtii grown under moderate to high light intensity $\left(400 \mu \mathrm{mol}_{\mathrm{ph}} \mathrm{m}^{-2} \cdot \mathrm{s}^{-1}\right)$. The aim is twofold: (a) to establish a list of reliable housekeeping genes valid for quantitative comparisons across several combinations of different wavelengths and trophic strategies and (b) to enable the investigation of the response of central carbon metabolic pathways under these process conditions. White, blue and red light emitting diodes (LEDs) were used to grow $\mathrm{pH}$ controlled photo- and mixo-trophic cultures over a period of $136 \mathrm{~h}$ in batch mode. A panel of 10 candidate genes, along with biomass growth rate and pigment content were dynamically monitored across all conditions. Statistical analysis identified genes (acetyl-CoA carboxylase subunit $\alpha$ and photosystem I reaction centre subunit II) with less variability observed in their expression levels across the entirety of conditions evaluated compared to housekeeping genes often referred to in literature (receptor of activated protein kinase $C$ and ribosomal protein (large subunit) 19). Further analysis of gene expression profiles revealed substantial differences in response to changes in both wavelength selection (upregulation of ribulose bisphosphate carboxylase small subunit under red phototrophic growth) and trophic strategy (upregulation of malate synthase from phototrophic to mixotrophic conditions). The systematic approach used to establish reliable reference genes presented herein enables robust comparisons of cellular responses across different conditions to better understand algal metabolism and improve process performance.
\end{abstract}

\section{Introduction}

Freshwater microalgae, like Chlamydomonas reinhardtii, have evolved an extensively versatile metabolism that increases their chances of survival in diverse environmental conditions in the presence (mixotrophy) or absence (autotrophy) of a readily available source of nutrients [1]. Due to this metabolic plasticity, and despite significant research efforts, our knowledge of algal metabolism remains nascent, relative to other crops, and our control over biomass composition remains rudimentary [2]. One of the main reasons that fully understanding algal metabolism and how it responds to varying environmental and bioprocessing conditions is far from trivial is the uniqueness of incident light as a critical process parameter. The effects of light on microalgal physiology are mediated by a variety of specialised, light harvesting and light sensing molecules. Chlorophylls, carotenoids, rhodopsins, phototropins and cryptochromes each have a unique absorption spectrum and are involved in different cellular processes like photosynthesis, phototaxis [3] or cell cycle control [4,5].

In addition, light in and of itself is complex in nature as it can vary in terms of intensity, photoperiod and spectral composition. Light intensity has a well documented and characterised positive effect on biomass growth both under phototrophic and mixotrophic conditions [6]. However, excessively high light intensities have been shown to trigger stress responses, like carotenoid production, in a number of algal species $[7,8]$ or even completely inhibit growth (photoinhibition) [9]. Phototrophic studies on biomass growth of $C$. reinhardtii have shown that below saturating light levels $\left(25-100 \mu \mathrm{mol}_{\mathrm{ph}} \mathrm{m}^{-2} \mathrm{~s}^{-1}\right)$, illumination with red and red-blue light can achieve a higher biomass yield on

\footnotetext{
* Corresponding author.

E-mail address: alex.kiparissides@ucl.ac.uk (A. Kiparissides).
} 
photons compared to white light [10]. However, at or above saturating light intensities $\left(>1500 \mu \mathrm{mol}_{\mathrm{ph}} \mathrm{m}^{-2} \mathrm{~s}^{-1}\right.$ ) the yield of biomass on photons was found to be inversely proportional to the specific photon absorption rate. Consequently, illumination at wavelengths considered to be suboptimal due to their relatively low specific absorption rate (e.g. illumination with yellow and warm white light) has been found to yield higher biomass yields than red or blue light at high light intensities [11].

The advent of light emitting diodes (LEDs) has enhanced our ability to accurately control several properties of incident light, beyond intensity, and investigate their effects on biomass growth and biochemical composition [12]. This has enabled in depth investigations of the effects of the frequency and duration of light/dark cycles, spanning timescales from milliseconds to hours (diel-cycle) [13-15]. Studies on the impact of light quality on biomass composition, primarily focused on pigment and/or lipid content in specialised producer species like Haematoccocus. pluvialis and Phaeodactylum tricornutum [16], have shown a variety of possible responses across species. Therefore, there is a need to understand the diverse and complex effects of the spectral composition of incident light on microalgal physiology and metabolism.

Associating gene expression profiles with changes in microalgal physiology under narrow band illumination can help unravel the specific effects of discrete regions of the photosynthetically active radiation (PAR) region of the visible light spectrum (400-750 nm). However, relative quantification of reverse transcription-quantitative real time polymerase chain reaction (RT-qPCR) measurements requires the existence of a set of reference genes with stable expression profiles across a variety of culture conditions. A number of studies have previously proposed candidate reference genes for $C$. reinhardtii with no clear consensus available in the literature due to the limited number of experimental conditions examined in each study [17-19]. A comprehensive study of the stability of four candidate reference genes under nitrogen deprivation both in photoautotrophic and mixotrophic conditions was recently published [20], however is limited to only white light illumination. An extensive transcriptomic analysis of $C$. reinhardtii gene expression under a variety of nutrient depletion conditions has been recently published [21].

Given the biotechnological potential of triggering desired responses in a microalgal culture by utilising a tailored spectral composition, this study investigates the effects of wavelength selection and trophic strategy on gene expression in C. reinhardtii. The genetic responses elicited by monochromatic LEDs with narrow peak intensities at the two extremes of the PAR range (Blue, 440-480 nm and Red, 640-670 nm) under phototrophic and mixotrophic conditions were compared against white light control cultures. A set of 10 candidate reference genes was compiled from literature and their stability was assessed across all 6 experimental conditions. The novel reference genes identified herein, coupled with dynamic biomass and pigment content measurements, were used to evaluate the effect of wavelength and trophic strategy induced changes at key metabolic nodes. Finally, the expression levels of promoter genes and/or $5^{\prime}-/ 3^{\prime}$-untranslated regions $\left(5^{\prime}-/ 3^{\prime}\right.$-UTR) previously targeted for the expression of recombinant proteins in C. reinhardtii were characterised to discern favourable illumination conditions and trophic strategies.

\section{Materials and methods}

\subsection{Organism and growth conditions}

Chlamydomonas reinhardtii wild-type strain $11 / 32 \mathrm{C}$ was obtained from the Culture Collection of Algae and Protozoa (CCAP, Scotland). C. reinhardtii 11/32C was grown in M8a media [22] supplemented with $7.49 \mathrm{mM}\left(\mathrm{g} \cdot \mathrm{L}^{-1}\right)$ of ammonia as the Nitrogen source [23]. Mixotrophic cultures were grown in modified M8a medium (referred to as M8a.Ac)

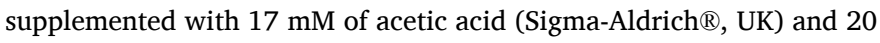
$\mathrm{mM}$ Tris base (Sigma-Aldrich ${ }^{\circledR}, \mathrm{UK}$ ). Seed cultures were inoculated from streaked agarose plates in $500 \mathrm{~mL}$ of M8a medium and grown in an
ALGEM Environmental Modelling Labscale Photobioreactor (Algenuity, $\mathrm{UK}$ ) at $25^{\circ} \mathrm{C}$ with $\mathrm{pH}$ controlled at $7 \pm 0.1$. Incident light intensity for all seed cultures was linearly increased during the first $48 \mathrm{~h}$ until 400 $\mu \mathrm{mol}_{\mathrm{ph}} \mathrm{m}^{-2} \cdot \mathrm{s}^{-1}$ and was subsequently kept constant for up to $84 \mathrm{~h}$. Cultures for the experimental work presented herein were inoculated

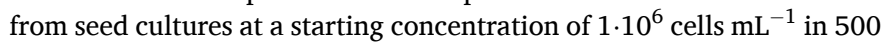
$\mathrm{mL}$ of either M8a or M8a.Ac media and maintained under constant illumination at $400 \mu \mathrm{mol}_{\mathrm{ph}} \mathrm{m}^{-2} \cdot \mathrm{s}^{-1}$ throughout the experiment. The experiments were performed at $25{ }^{\circ} \mathrm{C}$, under continuous aeration $(75$ $\mathrm{cm}^{3} \mathrm{~min}^{-1}$ ) provided through an HD-601 aquarium pump (Hidom, China). $5 \% \mathrm{CO}_{2}$-enriched air was automatically supplied on demand (75 $\mathrm{cm}^{3} \mathrm{~min}^{-1}$ ) to maintain $\mathrm{pH}$ at $7 \pm 0.1$. In the remainder of the text, the following abbreviations are used to describe wavelength selection (W: white light, B: blue light 440-480 nm, R: red light 640-670 nm) and trophic conditions (A: phototrophic growth in M8a, X: mixotrophic growth in M8a.Ac).

\subsection{Chlorophyll quantification}

$1 \mathrm{~mL}$ samples were collected post inoculation and after $48 \mathrm{~h}$ of culture. The samples were spun down at for $2 \mathrm{~min}$ at $5000 \mathrm{~g}$ (Eppendorf 5415, Eppendorf, UK) under constant temperature $\left(4^{\circ} \mathrm{C}\right)$. The supernatant was aspirated, and pigments were extracted by re-suspending the cell pellet in $1 \mathrm{~mL}$ of dimethylformamide (DMF, Sigma-Aldrich ${ }^{\circledR}, \mathrm{UK}$ ) while avoiding direct exposure to light. The resuspended pellets were centrifuged for $2 \mathrm{~min}$ at $10,000 \mathrm{~g}$ under constant temperature $\left(4^{\circ} \mathrm{C}\right)$ and the supernatant was transferred to a solvent resistant BRAND ${ }^{\circledR}$ UV cuvette (Merck, Germany). Chlorophylls A and B were quantified through absorbance readings at 647 and $664 \mathrm{~nm}$ respectively according to their maximum absorption coefficients [24].

\subsection{Cell size distribution}

Cell size distribution was measured with a CASY ${ }^{\circledR}$ TTC Cell Counter and Analyser System (Omni Life Science, Germany) as previously described [25]. Briefly, $50 \mu \mathrm{L}$ of culture were diluted in $10 \mathrm{~mL}$ of Casyton $₫$ buffer (Omni Life Science, Germany), mixed by inversion and immediately processed.

\subsection{Ribonucleic acid (RNA) extraction}

Samples containing $5 \cdot 10^{6}$ cells were centrifuged for $5 \mathrm{~min}$ at $12,000 \mathrm{~g}$ (Eppendorf 5415, Eppendorf, UK) under constant temperature $\left(4^{\circ} \mathrm{C}\right)$. The supernatant was aspirated and discarded, and the cell pellet was stored at $-80{ }^{\circ} \mathrm{C}$ for up to 4 months until further processing. RNA extraction was performed in $2 \mathrm{~mL}$ MaXtract High Density Tubes (Qiagen, Netherlands) using TRIzol ${ }^{\circledR}$ (Invitrogen, USA) and following the manufacturer's instructions. GlycoBlue ${ }^{\mathrm{TM}}$ Coprecipitant (Invitrogen, USA), dissolved in $50 \mu \mathrm{L}$ of RNase-free TE buffer (Sigma-Aldrich $\AA$, UK), was used to stain the nucleic acid pellet. Contaminating gDNA was removed with RNase-free DNase (Qiagen, Netherlands) according to the manufacturer's protocol. RNA integrity was assessed with a $1 \%$ ethidium bromide stained agarose gel in TBE buffer. RNA purity and concentration were assessed using a NanoDrop spectrophotometer (Thermo Fisher, USA). Ratios of absorbance readings at 260/280 nm and $230 / 260 \mathrm{~nm}$ ratios displayed little variability $(2 \pm 0.1)$ and indicated a lack of phenol contamination from the TRIzol based extraction.

\subsection{Primer design}

Gene primers for RT-qPCR were designed following the Minimum Information for Publication of Quantitative Real-Time PCR Experiments (MIQE) guidelines. Gene sequences were obtained from the Chlamydomonas reinhardtii genome v5.5 in the Phytozome database [26].A custom set of parameters prioritising primer pairs that span intron-exon junctions was used to select primer sequences in primer3 [27]. Primer 
quality was assessed with PCR Primer Stats [28]. Melt curve analysis with no template controls (NTC), no reverse transcription controls (NRT) and positive controls was used to ensure each primer pair gave rise to a single, well-defined peak from extracted RNA. Serial and linear dilutions were used to establish the log-linear dynamic range for each primer pair and primer efficiency. Starting RNA and primer mix concentrations were optimised over three orders of magnitude (1.5-150 ng $\mu \mathrm{L}^{-1}$ and 0.9-90 $\mu \mathrm{M}$ respectively).

\subsection{Quantitative reverse transcription polymerase chain reaction ( $R T$ - $q P C R)$}

RT-qPCR was carried out with a Luna ${ }^{\circledR}$ Universal One-Step RT-qPCR Kit (New England Biolabs, USA) on a CFX 96 connect Real-Time PCR System (Bio-Rad, USA). Individual RT-qPCR reactions had a total volume of $20 \mu \mathrm{L}$ made up of $10 \mu \mathrm{L}$ Luna Universal One-Step Reaction Mix $(2 \times), 1 \mu \mathrm{L}$ Luna WarmStart ${ }^{\circledR} \mathrm{RT}$ Enzyme Mix $(20 \times), 2 \mu \mathrm{L}$ forward and reverse primer mix $(9 \mu \mathrm{M}), 5 \mu \mathrm{L}$ RNA (75 ng) and $2 \mu \mathrm{L}$ Nuclease-Free Water. Thermal cycling was programmed according to the kit manufacturer's instructions. In short, 1 cycle of reverse transcription at $55^{\circ} \mathrm{C}$ for $10 \mathrm{~min}$ followed by 1 cycle of initial denaturation at $95^{\circ} \mathrm{C}$ for $1 \mathrm{~min}$ and 40 cycles of denaturation and extension at $95^{\circ} \mathrm{C}$ (for $10 \mathrm{~s}$ ) and $60^{\circ} \mathrm{C}$ (for $30 \mathrm{~s}$ ) respectively. A $60-95^{\circ} \mathrm{C}$ melt curve was generated to evaluate primer specificity and gDNA contamination.

\subsection{Reference gene selection and statistical analysis}

A panel of 10 candidate reference genes was compiled from literature (Table 1). A set of six diverse experiments including combinations of three different wavelengths and two different trophic strategies was designed to evaluate stability in expression levels. Expression levels of the candidate genes (Table 1 ) were measured by RT-qPCR with samples taken 16, 48 and $136 \mathrm{~h}$ hours post inoculation and analysed with the qBase+ software package (Biogazelle). The geNorm algorithm [29,30] was used to calculate the coefficient of variation of the normalised reference gene expression levels (CV) and the geNorm stability M-value (M) was used to rank candidate reference genes from least to most stable. Gene expression data were further analysed by performing $3(\mathrm{~W}, \mathrm{R}$, B) by $2(\mathrm{~A}, \mathrm{X})$ factorial ANOVAs to evaluate main and interaction effects of wavelength and trophic strategy on the average relative expression of each gene of interest. Multiple comparisons with Benjamini-Hochberg correction were performed in the statistical software R (version 3.6.1).

\section{Results}

The scientific literature is rich with studies on the phenotypic and metabolic response of $C$. reinhardtii to different intensities and qualities of light. Specifically in terms of light intensity, studies have been conducted under low $\left(10-100 \mu \mathrm{mol}_{\mathrm{ph}} \mathrm{m}^{-2} \cdot \mathrm{s}^{-1}\right)$ [20], moderate (100-700 $\left.\mu \mathrm{mol}_{\mathrm{ph}} \mathrm{m}^{-2} \cdot \mathrm{s}^{-1}\right)$ [31,32] or high $\left(>700 \mu \mathrm{mol}_{\mathrm{ph}} \mathrm{m}^{-2} \cdot \mathrm{s}^{-1}\right)$ [11,33,34] light intensities. In recent years, studies with a focus on industrial applications such as the production of speciality chemicals or the expression of recombinant proteins have focused on moderate and high light intensities [11,33,34]. In the present study, a moderate light intensity of $400 \mu \mathrm{mol}_{\mathrm{ph}} \mathrm{m}^{-2} \mathrm{~s}^{-1}$ was used for all culture conditions. Consequently, caution should be used when extrapolating the conclusions discussed below to significantly higher or lower light intensities.

\section{Table 1}

List of candidate reference genes monitored in this study with RT-qPCR (ATPD, ATP synthase delta chain, chloroplastic; $R P L-19$, ribosomal protein (large subunit) 19; RACK1, receptor of activated protein kinase C; RPS-10, ribosomal protein (small subunit) 10; ch3-II, histone H3; psaD, photosystem I reaction centre subunit II; ACX1, acetyl-CoA carboxylase subunit $\alpha$; PSRP-1, plastid specific ribosomal protein(small subunit) 1; STA1, ADP-glucose pyrophosphorylase large subunit 1; DLA3, dihydrolipoamide acetyltransferase component of pyruvate dehydrogenase; RBCS, ribulose bisphosphate carboxylase small subunit; FUM1, fumarate hydratase; MAS1, malate synthase; KAS2, 3-oxoacyl-[acyl-carrier-protein] synthase; atpA, ATP synthase subunit alpha; HSP70A, heat shock protein 70A).

\begin{tabular}{|c|c|c|c|c|c|}
\hline Gene & Gene name & Forward Primer $\left(5^{\prime}-3^{\prime}\right)$ & Reverse Primer $\left(5^{\prime}-3^{\prime}\right)$ & $\begin{array}{l}\text { Used as reference } \\
\text { gene in }\end{array}$ & Phytozome ID \\
\hline$A T P D$ & ATP synthase delta chain, chloroplastic & GTTCCTGATCGCCAAGAAGC & GAGTCAATCACGGGCTTCAG & This study & $\begin{array}{l}\text { Cre11.g467569. } \\
\text { t1.1 }\end{array}$ \\
\hline RACK1 & Receptor of activated protein kinase $C$ & AAGACCATCAAGCTGTGGAACA & TTCCAGACCTTGACCATCTTGT & Siaut07,Beel12 & $\begin{array}{l}\text { Cre06.g278222. } \\
\text { t1.1 }\end{array}$ \\
\hline RPS-10 & Ribosomal protein (small subunit) 10 & CTGGTGACTGAGCGCTTCT & GGGCGGTTGGACTTCTTCA & Siaut07,Liu12 & $\begin{array}{l}\text { Cre09.g411100. } \\
\text { t1.2 }\end{array}$ \\
\hline psaD & Photosystem I reaction centre subunit II & GACACTCCCAGCCCGATTTTC & CCAGGTGATGACGTAGAACTCC & This study & $\begin{array}{l}\text { Cre05.g238332. } \\
\text { t1.1 }\end{array}$ \\
\hline$A C X 1$ & Acetyl-CoA carboxylase subunit $\alpha$ & AGCAAGACTCTGGTTAGCGATG & CCCAAAGCGAGACAGGATAGTG & This study & $\begin{array}{l}\text { Cre12.g519100. } \\
\text { t1.2 }\end{array}$ \\
\hline PSRP-1 & Plastid specific ribosomal protein(small subunit) 1 & GCAAGAAGGAGCAGAAGGTAGA & TCCTTGATCTTCTGGAGCTTGG & This study & $\begin{array}{l}\text { Cre05.g237450. } \\
\text { t1.2 }\end{array}$ \\
\hline STA1 & ADP-glucose pyrophosphorylase large subunit 1 & CTCGGTGCTGTCCATCATTCT & GTTCAGGGAGGTCGAGTTGAA & This study & $\begin{array}{l}\text { Cre13.g567950. } \\
\text { t1.2 }\end{array}$ \\
\hline FUM1 & Fumarate hydratase & AGAACTGCATCAAGAAGGTGGA & AGAAGTCGTTACCCTTGTCGTC & NA & $\begin{array}{l}\text { Cre06.g254400. } \\
\text { t1.1 }\end{array}$ \\
\hline MAS1 & Malate synthase & CCCAACGGCAAGGTCTACAG & CAGCGTTGTGGAAGAAGAACAG & NA & $\begin{array}{l}\text { Cre03.g144807. } \\
\text { t1.1 }\end{array}$ \\
\hline KAS2 & 3-oxoacyl-[acyl-carrier-protein] synthase & CTCACAAAGTTTCCTCGGCAAG & CGCCTTCTCAGTCTTGGAAATG & NA & $\begin{array}{l}\text { Cre07.g335300. } \\
\text { t1.2 }\end{array}$ \\
\hline $\operatorname{atp} A$ & ATP synthase subunit alpha & GCAATGCGTACTCCAGAAGAAC & CGAGCAATACCGTCACCTACTT & NA & $2717041^{\mathrm{d}}$ \\
\hline HSP7OA & Heat shock protein $70 \mathrm{~A}$ & CCAAGAACCAGGTCGCTATGAA & ACCTTCTCCTCGTTCTTGTAGG & NA & $\begin{array}{l}\text { Cre08.g372100. } \\
\text { t1.2 }\end{array}$ \\
\hline
\end{tabular}




\subsection{Expression stability}

Relative quantification of gene expression via RT-qPCR requires the determination of one (or more) reference genes which are expected to maintain a stable expression level across all experimental conditions examined [35]. The panel of candidates genes (Table 1) investigated in the present study reflects three distinct selection criteria: (a) genes extensively used as reference genes in relevant scientific literature (RACK1, RPL19, CH3-I and RPS10 [19,36-39]), (b) genes with functional similarities to widely used reference genes (PSRP-1, psaD and $A T P D[40]$ ) and, novel in this study, (c) genes involved in central carbon metabolism due to its vital role in cellular function under most relevant experimental conditions (DLA3, ACX1 and STA1). Gene expression was measured at three time points $(16,48$ and $136 \mathrm{~h}$ post inoculation) for each of the six possible combinations of wavelength (W, R, B) and trophic strategy (A, X).

Raw RT-qPCR data is evaluated in terms of quantification cycle $\left(C_{q}\right)$, which is the number of amplification cycles required for a RT-qPCR reaction's fluorescence to surpass a defined threshold. This is inversely correlated to the specific cDNA template present in the reaction. The 10 genes evaluated displayed a wide range of average $\mathrm{C}_{\mathrm{q}}$ values $\left(\overline{C_{q}}\right)$ with $A T P D$ at the low end $\left(\overline{C_{q}}=14\right)$ and DLA3 with at the high end of the range $\left(\overline{C_{q}}=22\right)$. An overview of all $\mathrm{C}_{\mathrm{q}}$ ranges measured for candidate reference genes under all experimental conditions examined in this paper is presented in Fig. 1. Interestingly the $\mathrm{C}_{\mathrm{q}}$ value for $A T P D$ was also found to have lowest variability across all conditions while the $\mathrm{C}_{\mathrm{q}}$ values for PSRP-1, chosen for its similarity to canonical ribosomal protein genes utilised as reference genes throughout literature, varied significantly between conditions. Given its exclusively plastidic localisation, it is possible that ribosomal activity in the chloroplast is, at least partially, regulated at the transcriptional level in response to changes in the quality of incident light.

Prior to the development of sophisticated data analysis algorithms and the widespread availability of real-time PCR systems, relative quantification was primarily based on a single reference gene derived either from literature or through simple, yet effective, log-linear models $\left(\Delta \Delta \mathrm{C}_{\mathrm{q}}\right)[41,42]$. However, current state of the art for comparison of gene expression across widely varying conditions or cell lines involves the use of sophisticated algorithms that employ two or more genes to normalise gene expression measurements [43]. The geNorm algorithm, used in the

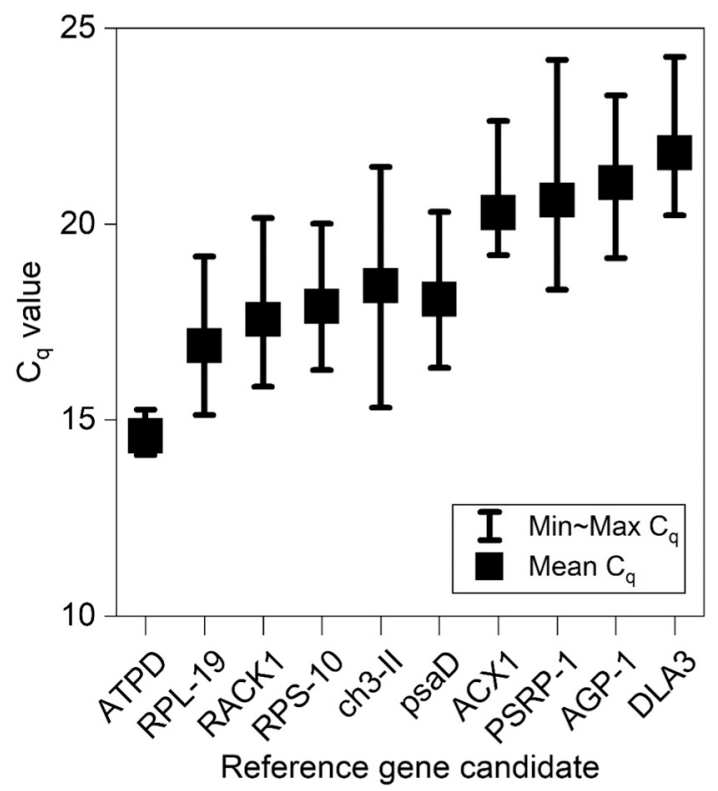

Fig. 1. Mean $\mathrm{C}_{\mathrm{q}}$ of candidate reference genes studied. ( $n=6$, error bars represent minimum and maximum $\mathrm{C}_{\mathrm{q}}$ ). present study, has been successfully applied to a wide variety of organisms $[18,44,45]$. The algorithm uses pairwise variation $(\mathrm{Vn} / \mathrm{n}+1)$ to evaluate the number of reference genes required to achieve an accurate normalisation factor $(\mathrm{M})$ which will remain stable across all experimental conditions and cell lines studied [29,30]. Analysis of the results indicated that $A C X 1$ and $p s a D$ form the most stable pair of genes with a $\mathrm{V}_{2 / 3}$ value lower than the recommended threshold $(<0.15)$ [29] indicating that additional reference genes are not required.

ACX1 encodes the $\alpha$-carboxyltransferase subunit of the multimeric acetyl coenzyme A (acetyl-CoA) carboxylase (ACCase) and has been shown to be expressed in co-ordination with the other subunits in Arabidopsis thaliana [46] and C. reinhardtii [47]. While significant variations in the expression levels of both the $\alpha$ - $[20,47]$ and the $\beta$ - [48] carboxyltransferase subunits have been reported in nitrogen starved cells, expression levels of $A C X 1$ have been shown to remain stable under nitrogen replete conditions, in agreement with the findings in the present study.

The $p s a D$ gene encodes subunit II of photosystem I (PSI), an essential component for PSI accumulation in Arabidopsis thaliana [49] and key to the efficient functioning of PSI in Synechocystis sp [50]. A highly expressed constitutive gene, its promoter element has always remained relevant in recombinant protein expression studies in $C$. reinhardtii $[51,52]$. Light intensity and light quality have been shown to affect the pigment content and composition of microalgal cells but, to the extent of our knowledge, none of the available studies tested the expression levels of $p s a D$ under monochromatic illumination. It is not particularly surprising that a gene expressing an essential PSI subunit presents robust expression across the conditions studied in the present study as essential photosynthetic components are commonly controlled by posttranslational regulatory processes such as epistasy of synthesis (CES) [53].

\subsection{Growth and gene expression under different wavelengths and trophic strategies}

Batch cultures of $C$. reinhardtii $11 / 32 \mathrm{C}$ were grown for $136 \mathrm{~h}$ under constant illumination at three different wavelengths (W, R, B) using media with (X) and without (A) a readily available organic carbon source. Daily biomass measurements (OD $750 \mathrm{~nm}$ ) were used to estimate growth rate during the exponential growth phase ( $\mu_{\text {exp }}$, Table 2, Fig. S1). In agreement with findings from other studies [11], white light illumination was found to have the highest growth rate in both trophic strategies. Interestingly, under the constant environmental conditions considered in the present study $(\mathrm{pH}$, temperature and light intensity), the presence of an organic carbon source had a significant impact on biomass growth only under blue light illumination. In contrast, the addition of a carbon source under white light illumination (WX) resulted in $5 \%$ reduction in $\mu_{\exp }$ compared to (WA). The rate of acetate consumption in mixotrophic cultures $\left(q_{A c}\right.$, Table 2$)$ was nearly identical under all studied wavelengths irrespective of the observed biomass growth rate. Acetate was fully extinguished from the media within the first $48 \mathrm{~h}$ post inoculation in all mixotrophic conditions.

\section{Table 2}

Summary of macroscopic growth parameters of $C$. reinhartdtii cultured under different illumination and trophic strategies. Values calculated based on biomass and acetate concentrations between $\mathrm{t}_{1}=0 \mathrm{~h}$ and $\mathrm{t}_{2}=48 \mathrm{~h}$. W: white LED light; R: red LED light 640-670 nm; B: blue LED light 440-480 nm; A: phototrophic growth in M8a; X: mixotrophic growth in M8a.Ac; $\mu_{\text {exp }}$ : observed biomass growth rate (hour ${ }^{-1}$ ); $\mathrm{q}_{\mathrm{Ac}}$ : observed acetate uptake rate $\left(\right.$ hour $^{-1}$ ).

\begin{tabular}{llllllll}
\hline & & WA & RA & BA & WX & RX & BX \\
\hline$\mu_{\exp }\left(\mathrm{h}^{-1}\right)$ & Flask 1 & 0.076 & 0.054 & 0.042 & 0.064 & 0.055 & 0.056 \\
& Flask 2 & 0.069 & 0.056 & 0.040 & 0.074 & 0.058 & 0.050 \\
$\mathrm{q}_{\mathrm{Ac}}\left(\mathrm{mM} \mathrm{h}^{-1}\right)$ & Flask 1 & - & - & - & 0.363 & 0.369 & 0.370 \\
& Flask 2 & - & - & - & 0.361 & 0.370 & 0.370 \\
\hline
\end{tabular}


The expression levels of a panel of genes selected based on their metabolic function (Table 1) were measured and analysed for each of the six experimental conditions described above. All reported gene expression levels were calculated relative to the expression of the two most stable reference genes identified in Section 3.1 (ACX1 and psaD). Gene expression levels for central carbon metabolism genes were estimated by averaging values obtained from two samples taken during the exponential growth phase ( $16 \mathrm{~h}$ and $48 \mathrm{~h}$ ). The expression level of promoter genes was evaluated at three different time points ( $16 \mathrm{~h}, 48 \mathrm{~h}$ and $136 \mathrm{~h}$ ) throughout the culture. In the sections that follow, gene expression results are presented and discussed grouped by metabolic pathway.

\subsection{Photosynthesis}

The ribulose-1, 5-bisphosphate carboxylase oxygenase (RUBISCO) found in $C$. reinhardtii is a hexadecamer composed of eight small subunits (SS) and 8 large sub-units (LS). Two variants of the small subunit of RUBISCO have been identified in the Chlamydomonas nuclear genome (RBCS1 and RBCS2) [54]. While their peptide products differ by only four amino acids and are functionally redundant [54], their expression is dependent both on trophic strategy and the presence or absence of light $[55,56]$. The primer pair designed and used in this study (Table 1) amplifies both sub-unit variants ( $R B C S 1$ and $R B C S 2$ ) to enable the evaluation of overall $R B C S$ expression irrespective of individual subunit related dependences (Fig. 2A). Wavelength selection had a statistically significant effect $(p<0.01)$ on the average expression level of $R B C S$ with average expression levels under red light (R) being significantly higher compared to both white (W) and blue (B) light in both trophic conditions (A, X). In addition, the average $R B C S$ expression in cultures grown under blue light (B) was significantly higher $(p<0.05)$ than those grown under white light (W) in both trophic conditions. It is worth noting that the macroscopically observed biomass growth rate (Table 2) does not appear to be strongly correlated with RBCS expression levels. Under both trophic strategies, (R) illuminated cultures displayed statistically significant higher RBCS expression levels than (W) illuminated cultures, however resulted in lower growth rate values. Similarly, low levels of $R B C S$ expression measured in $\mathrm{B}$ and $\mathrm{W}$ cultures resulted in substantially different growth rates (Table 2). These differences were observed in both trophic conditions and the presence of an external carbon source did not seem to have a significant impact on $R B C S$

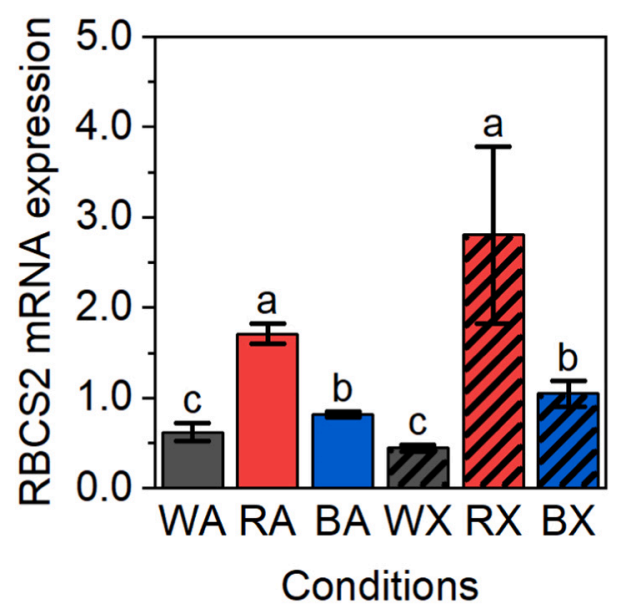

Fig. 2. Carbon fixation related gene expression in the exponential growth phase. Average Ribulose bisphosphate carboxylase small subunit (RBCS) gene expression from $16 \mathrm{~h}-48 \mathrm{~h}(n=4$, error bars represent SEM, different error bar labels indicate statistically significant differences between conditions, $p<$ 0.05). W: white light, R: red light $640-670 \mathrm{~nm}$, B: blue light $440-480 \mathrm{~nm}, \mathrm{~A}$ : phototrophic growth in M8a, X: mixotrophic growth in M8a.Ac. (For interpretation of the references to colour in this figure legend, the reader is referred to the web version of this article.) expression levels.

In order to further understand the implications of these results, cell size distribution during the mid-exponential growth phase $(48 \mathrm{~h})$ was measured for all conditions (Fig. 4). In both trophic conditions examined, cells grown under constant red light illumination had a smaller average diameter, while cells grown under constant blue light illumination had a larger average diameter when compared to control cultures grown under white light. The growth rate data (Table 2), calculated using optical density measurements $(750 \mathrm{~nm})$, coupled with the cell size distribution data (Fig. 4) indicate that wavelength selection has an impact on the cell cycle $[57,58]$. The balanced combination of long- and short-wavelengths making up white light results in the fastest biomass production under both phototrophic (WA) and mixotrophic (WX) conditions. Additionally, an average cell cycle length is maintained and consequently cell size remains in-between the smaller cells produced by (R) and the larger cells produced by (B) in both trophic strategies. The upregulation of $R B C S$ under red light indicates an increased need for energy generation, likely required to operate a shorter cell cycle and maintain a rapid duplication rate. On the other hand, cells grown under blue light had lower RBCS expression levels (Fig. 2A), a lower apparent growth rate (Table 2) and the largest observed average cell diameter (Fig. 4). These results suggest that blue light illumination leads to larger cells on average with an longer or arrested cell cycle while red light illumination leads to smaller, rapidly dividing cells. However, in terms of overall biomass yield (in gDCW/L) both extremes of the PAR spectrum underperform when compared to white light illumination.

Chlorophyll (Chl) a/b measurements (Fig. 3) revealed a statistically significant higher $\mathrm{Chl} \mathrm{a} / \mathrm{b}$ ratio in cultures grown under red light (RA, $\mathrm{RX}$ ). The $\mathrm{Chl} \mathrm{a} / \mathrm{b}$ ratio has been previously inversely linked to PSII antenna size due to Chl b's preference for binding to peripheral antenna complexes [59]. Moreover, in agreement with previous studies [10], the specific amount of Chl b per pg of biomass was significantly lower under red light illumination. Given that incident light intensity was kept
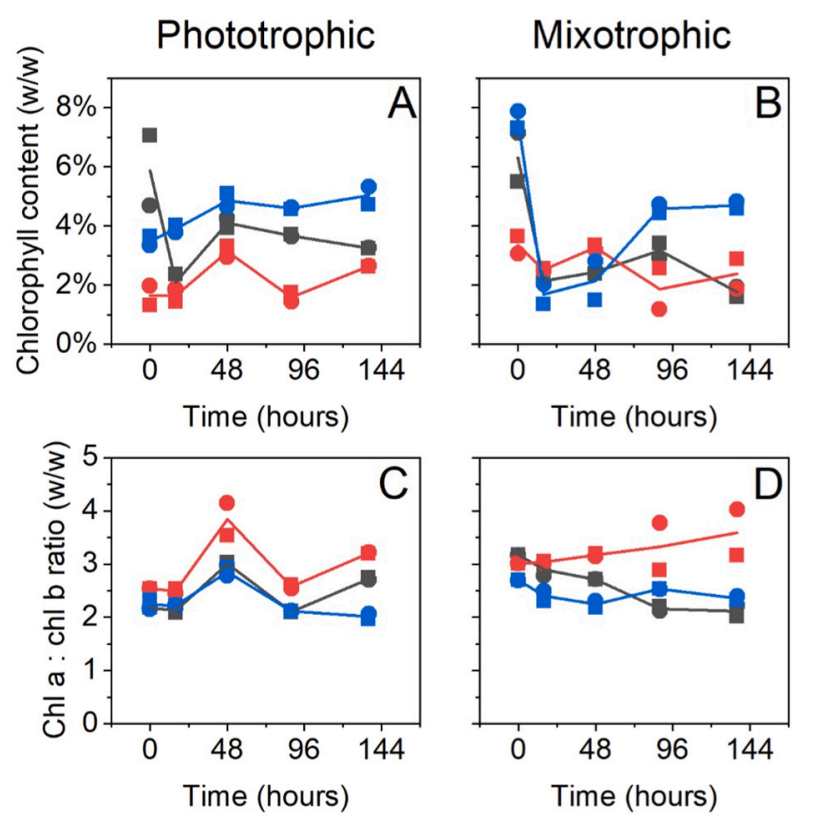

Fig. 3. Chlorophyll content and composition during an entire batch culture. (A, B) Chlorophyll content as a percentage of total biomass. (C,D) Chlorophyll a to chlorophyll $b$ ratio. The two biological replicates $(n=2)$ are indicated by squares (Flask 1) and circles (Flask 2) respectively. Solid lines indicate the average across the biological replicates for each condition. Black symbols and lines used for white light; Red symbols and lines used for red light 640-670 nm; Blue symbols and lines used for blue light 440-480 nm. (For interpretation of the references to colour in this figure legend, the reader is referred to the web version of this article.) 
constant across all experimental conditions, this further supports the hypothesis stated above, that red light illumination leads to a shortened cell cycle. High values of the $\mathrm{Chl} \mathrm{a} / \mathrm{b}$ ratio, coupled with a smaller antenna [59] and average cell size (Fig. 4) result in reduced surface area for absorption of photons and increased photosynthetic efficiency as well as reduced mutual shading across the light path. These effects combine to increase the proportion of cells in the culture receiving enough light to maintain a high growth rate. Consequently, the implied increase in light utilisation efficiency under red light illumination would result in an increased demand for Calvin-Benson cycle (CBC) enzymes like RUBISCO, and potentially increased gene expression as has been observed in the present study (Fig. 2A), to fulfil the increased demand. When considering the monochromatic conditions of this study in isolation, there is a strong positive correlation between biomass growth rate and $R B C S$ expression in the autotrophic conditions that is lost in the mixotrophic regime. This suggests that the availability of an organic carbon source and the resulting switch to mixotrophic metabolism reduce the relevance of photosynthetically generated metabolic resources in biomass growth rate maximisation. This is not apparent in growth under white light, possibly due to its more varied spectral distribution resulting in optimal phototrophic growth at a lower light utilisation efficiency compared to monochromatic illumination.

\subsection{Acetyl coenzyme A catabolism}

Acetate uptake rates (Table 2) were found to be independent of wavelength selection and biomass growth rate. However, when comparing biomass growth across trophic strategies for the same wavelength selection, differences in metabolic behaviour can be observed. In cultures grown under constant white light (WA, WX) and red light (RA, RX) illumination, the addition of acetate as a carbon source resulted in a relatively small change in biomass growth rate (Table 2). This suggests that under appropriately controlled conditions phototrophic growth under each of these two lights is nearly optimal. It seems that introducing an organic carbon source under these conditions does not result in an increased rate of biomass production. In contrast, for cultures grown under blue (BA, BX) light, the addition of acetate resulted in a substantial increase of the growth rate. Interestingly, $R B C S$ expression levels remained similar across the different trophic strategies (WA, WX, RA, RX and BA, BX; Fig. 2A), indicating no drastic change in the rate of carbon fixation from photosynthesis. Therefore, under white

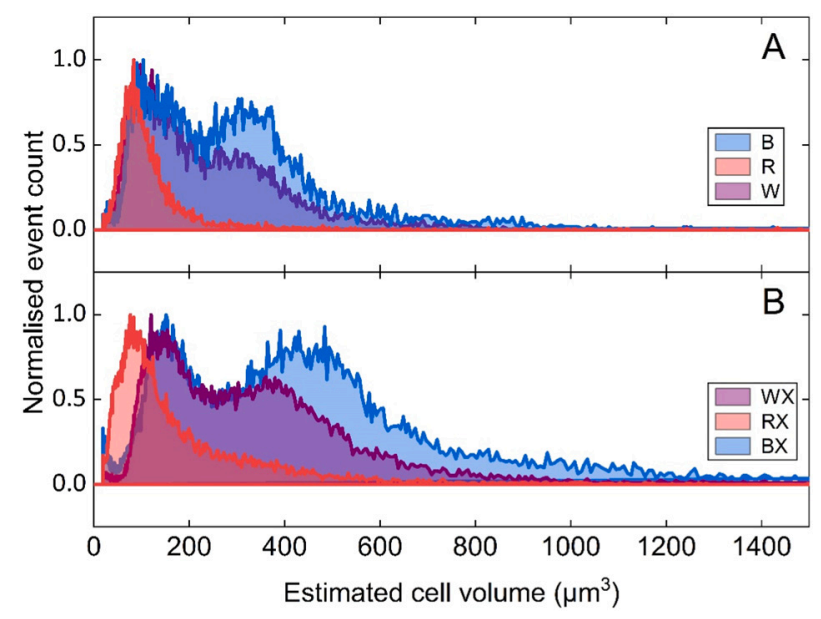

Fig. 4. Cell size distribution measured at $48 \mathrm{~h}$ in the exponential growth phase. (A) Representative cell size distribution of cells grown in phototrophic mode with M8a media. (B) Representative cell size distribution of cells grown in mixotrophic mode with M8a.Ac media. W: white light, R: red light 640-670 nm, B: blue light $440-480 \mathrm{~nm}$, A: phototrophic growth in M8a, X: mixotrophic growth in M8a.Ac. (For interpretation of the references to colour in this figure legend, the reader is referred to the web version of this article.) and red illumination the additional carbon uptaken in the form of acetate must be assimilated through a different metabolic pathway, one not directly linked to biomass growth. The presence of acetate is known to trigger a reconfiguration of $C$. reinhardtii metabolism as the inorganic carbon fixation of photosynthesis is complimented by the organic carbon assimilation of acetate via its conversion to acetyl-CoA [60].

In nutrient replete mixotrophic conditions acetyl-CoA is preferentially produced in a single enzymatic step catalysed by acetyl-CoA synthase (ACS) [61]. ACS homologs have been identified in several cellular compartments (ACS1 - cytosol ACS2 - plastid, ACS3 - glyoxysomes and mitochondria) $[62,63]$. Consequently, this metabolic plasticity enables the cells to repurpose assimilated acetate to drive different metabolic pathways by channelling it to the appropriate cellular compartments. As can be seen in Fig. 5A, Acetyl-CoA is primarily utilised in fatty acid chain formation (cytoplasm), in the glyoxylate cycle (glyoxysome) and in the TCA cycle (mitochondria). Differences in transcriptional activity between photoautotrophic and mixotrophic metabolism may elucidate the fate of acetate derived carbon assimilated during mixotrophic growth. Therefore, the expression level of enzymes exclusive to each of the three main metabolic pathways that consume acetyl-CoA was evaluated for all experimental conditions.

\subsubsection{Tricarboxylic acid cycle}

The TCA cycle produces reducing equivalents for oxidative phosphorylation and is a vital metabolic process in non-photosynthetic organisms which produce the bulk of their cellular ATP in mitochondria. In contrast mitochondrial respiration rates in nutrient replete phototrophic and mixotrophic conditions average approximately $10-15 \%$ of gross photosynthetic rates across many phyla of microalgae including chlorophytes like $C$. reinhardtii [64]. In fact, several genes exclusive to the TCA cycle have been reported as non-essential for the growth of photosynthetic organisms [65]. Consequently, the availability of an organic carbon source is not expected to have a significant impact on the activity of TCA genes. To verify the validity of the above hypothesis, the expression level of the TCA exclusive FUM1 gene, which encodes the enzyme fumarate hydratase that catalyses the reversible hydration of fumarate to malate (Fig. 5A) was measured across all experimental conditions.

The addition of acetate had no impact on FUM1 expression in cultures grown under white and red light (WA, WX and RA, RX; Fig. 5B). In fact, FUM1 expression levels were statistically similar across all four conditions (WA, WX, RA, RX; Fig. 5B). This indicates, as expected, that there is no correlation between biomass growth (Table 2) and TCA cycle activity (assessed through the expression levels of FUM1 in the present study) in the nutrient replete conditions considered herein. Wavelength selection on the other hand, had a statistically significant effect on the expression level of FUM1 (Fig. 5B). Specifically, FUM1 expression was significantly lower in phototrophic cultures grown under blue light (BA). Interestingly, in cultures grown under blue light (BA, BX; Fig. 5B) the addition of acetate in the media led to an increased average expression level for FUM1. The increased expression level under blue light corresponded to an increased biomass growth rate (BX, Table 2) indicating that the additional carbon might be channelled towards mitochondrial respiration and ATP synthesis [66]. The positive correlation between FUM1 expression and biomass growth rate for cultures grown under blue light suggests that in the presence of a readily available carbon source cellular growth could rely on TCA cycle activity and heterotrophy.

\subsubsection{Glyoxylate cycle}

Algae can preferentially utilize acetate as a carbon source due to the availability of an abbreviated version of the TCA cycle, sharing five of the eight TCA cycle enzymes, known as the glyoxylate cycle. This enables them to bypass the two decarboxylation steps found in the TCA cycle and enables the biosynthesis of macromolecules from simple $\mathrm{C}_{2}$ carbon compounds $[62,67]$. The preferential use of the glyoxylate cycle 

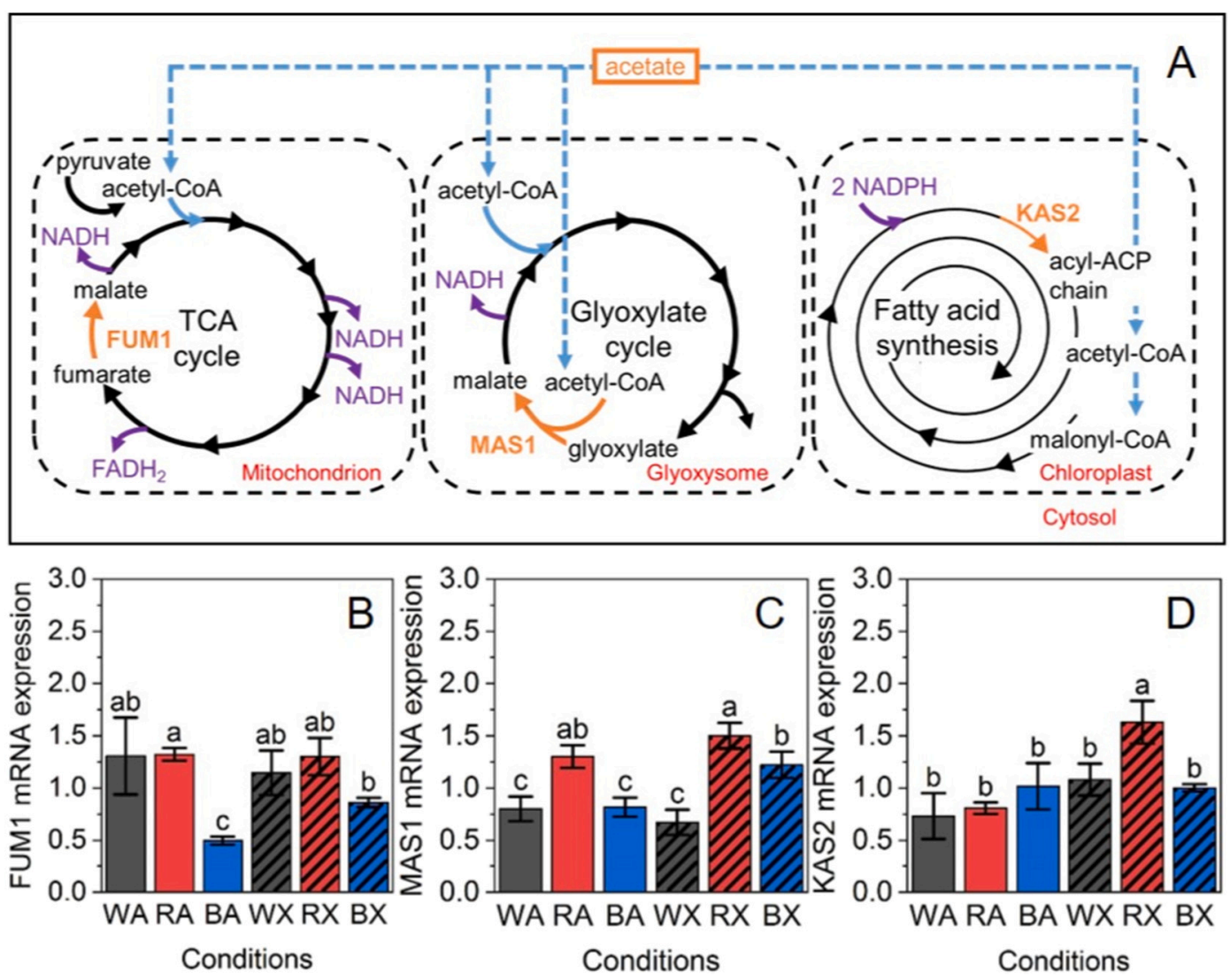

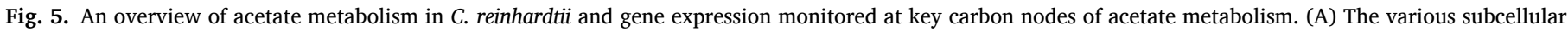

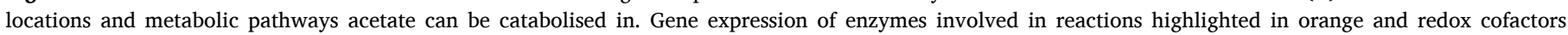

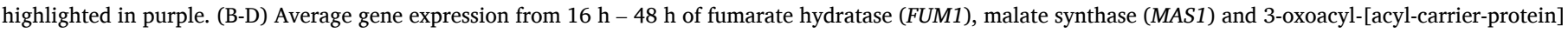

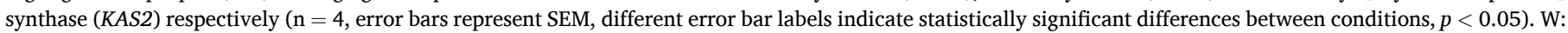

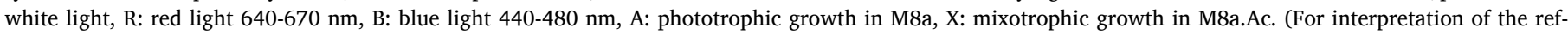
erences to colour in this figure legend, the reader is referred to the web version of this article.)

in the presence of a $\mathrm{C}_{2}$ organic acid has been recently investigated using a genome scale metabolic model of $C$. reinhardtii metabolism under nutrient replete mixotrophic and phototrophic conditions [68]. Although qualitative in nature, the results suggested a substantially increased flux through the glyoxylate cycle in the presence of a $\mathrm{C}_{2}$ organic acid. This theoretical model based approach was able to accurately predict the experimentally observed change in oxygen evolution rate between the two conditions [68]. In the present study, the expression of a glyoxylate cycle exclusive gene, MAS1, was measured across all experimental conditions. The MAS1 gene encodes malate synthase, an enzyme that catalyses the formation of malate $\left(\mathrm{C}_{4}\right)$ from glyoxylate $\left(\mathrm{C}_{2}\right)$ and acetyl-CoA $\left(\mathrm{C}_{23}-\right.$ Fig. 5A).

Surprisingly, the presence or absence of acetate had a statistically significant effect on MAS1 expression levels only in cultures grown under blue light (BA, BX; Fig. 5C). The 1.5-fold upregulation $(p<0.01)$ of MAS1 in mixotrophic cultures grown under blue light (BX) did not result in an increased biomass growth rate (Table 2). In conjunction with the observed upregulation of the TCA exclusive FUM1 in mixotrophic growth conditions (BA, BX; Fig. 5C), these results seem to indicate that carbon derived from acetate can help supplement biomass growth rate in non-optimal light conditions. No significant change in MAS1 expression levels was detected between phototrophic and mixotrophic conditions for cultures grown under white and red light (WA, WX and RA, RX; Fig. 5C). This is in contrast to previous studies [20,69] where an upregulation of glyoxylate cycle genes was observed following the addition of acetate. One possible explanation is that MAS1 expression levels are not linked to flux through the glyoxylate cycle in non-growth inhibited, constantly illuminated cultures (WA, WX, RA, RX) due to regulation occurring primarily by enzyme phosphorylation [70]. In fact, no correlation between growth (Table 2) and MAS1 expression (Fig. 5C) was found for cultures grown under white and red (WA, WX and RA, RX) light in the present study. Finally, wavelength selection was found to have a significant effect on MAS1 expression levels (Fig. 5C), with cultures grown under red light having a higher average expression level $(p$ $<0.05$ ).

\subsubsection{Fatty acid synthesis}

Acetyl-CoA derived from assimilated acetate in mixotrophic cultures can be channelled towards fatty acid biosynthesis which is driven by the heterogeneous fatty acid synthase (FAS) enzyme complex in the chloroplast [71]. The fatty acids generated can increase the cell's intracellular free fatty acid (FFA) pool or they can be incorporated into one of the many lipid classes produced by C. reinhardtii [72]. A multi-omics study found that protein levels of enoyl-ACP reductase were reduced during nitrogen deprivation, alongside a transient decrease in all FAS complex related transcripts during the first 12-48 h [21]. However, in the nutrient replete conditions of this study, where highly differing growth rates were observed between conditions it would be interesting to follow the gene expression of FAS complex related transcripts as a preliminary indicator of changes in macromolecular composition at a cellular level and in the assimilation of acetate derived carbon. The gene KAS2 encodes 3-ketoacyl-ACP synthase (KAS), the third enzyme in the FAS complex, which adds two carbons per FAS-cycle to the growing acyl-ACP chain using malonyl-ACP as its substrate (Fig. 5A).

Trophic strategy selection had an impact on KAS2 expression level in cultures illuminated under white and red light (WA, WX and RA, RX; 
Fig. 5D). In both cases, KAS2 expression was upregulated in the presence of acetate in the media, though the change in expression level was statistically significant only for cultures grown under red light $(p<0.05)$. The highest KAS2 expression level was observed in mixotrophic cultures grown under red light (RX; Fig. 5D), while KAS2 expression levels remained constant across cultures grown in white (WX) and blue (BX) light. In contrast, KAS2 expression levels remained constant, irrespective of trophic strategy, in cultures grown under blue light (BA, BX; Fig. 5D).

This suggests that under blue light, an increase in fatty acid production is not required to achieve the observed increase in biomass growth rate (Table 2). On the other hand, there was no change in biomass growth rate observed between trophic strategies in cultures grown under red light (R, Table 2). Meanwhile a statistically significant increase in KAS2 expression was observed in red light mixotrophic cultures (RX; Fig. 5D). One possible explanation could be that in cultures grown under red light with a smaller average cell size (Fig. 4) but otherwise comparable biomass growth rate (Table 2), the assimilated acetate in the form of acetyl-CoA is preferentially funnelled towards fatty acid production. This would lead to an increased production rate for membrane-lipids, necessary to support the shorter cell-cycle with more cell divisions per unit time for cells grown under red light (Fig. 4). Previous studies have shown that the autophagous degradation of membrane lipids for TAG production under nitrogen starvation is less pronounced in mixotrophic C. reinhardtii cultures grown in white light [73] further hinting at an increased flux of acetyl-CoA towards fatty acid synthesis during mixotrophy.

The gene expression data discussed in the preceding sections is summarised in Fig. 6. Mixotrophic cultures grown under constant red light illumination had the highest average fold expression for all genes considered thus far. However, this did not translate directly into an increased biomass growth rate as could have been expected given the nature of the genes investigated. This indicates that despite similarities in the observed biomass growth rates across conditions considered herein (Table 2), the macromolecular composition of cells will change depending on the utilised trophic and illumination strategy.

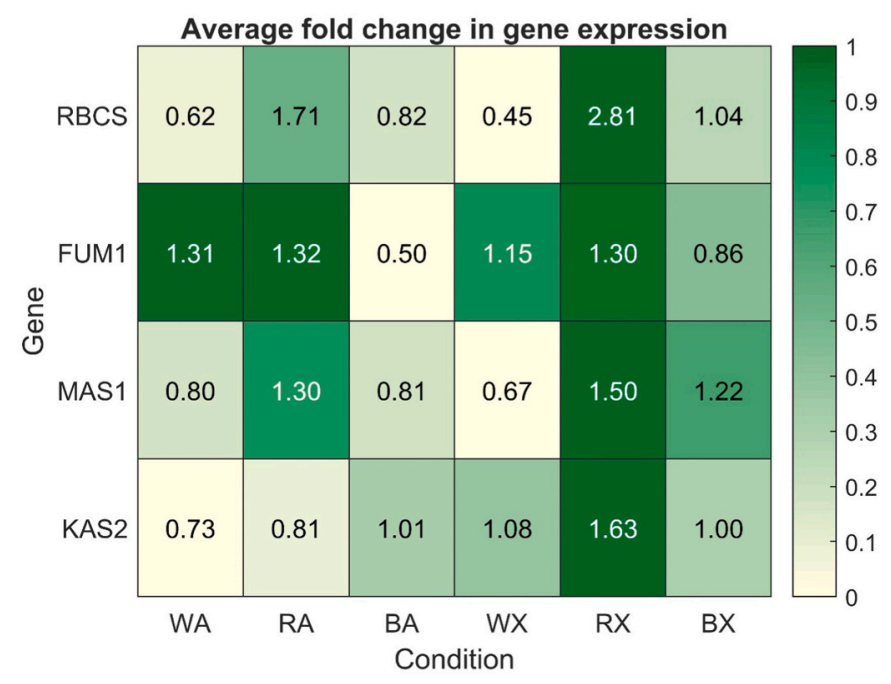

Fig. 6. Summary of average gene expression level compared across multiple light and media conditions. The colour scale is normalised in each row as the data reflects average fold change in gene expression and is not directly comparable between genes $(n=4)$. W: white light, R: red light 640-670 nm, B: blue light 440-480 nm, A: phototrophic growth in M8a, X: mixotrophic growth in M8a.Ac. (For interpretation of the references to colour in this figure legend, the reader is referred to the web version of this article.)

\subsection{Recombinant protein promoter genes}

In recent years, a number of sophisticated genome editing technologies have been adapted to microalgae [74]. C. reinhardtii, often referred to as the model microalgae, has the most complete algal genome editing toolbox however genetic engineering techniques are being developed for other type of algae as well $[75,76]$. Recombinant protein expression in $C$. reinhardtii has been attempted by integration of the recombinant genes into either the chloroplast [77] or the nuclear genome [78]. Each approach has its own benefits and limitations, with no clear consensus on an optimal strategy due to a lack of bioreactor (or larger) scale studies. As algal genome editing technologies mature, process development for recombinant protein expression will have to explore candidate promoters and culture conditions that lead to optimal transcriptional activity. In order to understand the magnitude of the impact process conditions can exert on transcriptional activity, the expression of a series of genes with promoters and/or $5^{\prime} / 3^{\prime}$ UTR(s) that have been previously used to facilitate recombinant gene expression in C. reinhardtii (Table 1) was evaluated across all experimental conditions. Bearing in mind that transcription and translation do not necessarily correlate well, some high level conclusions can be drawn from the data presented below.

\subsubsection{Ribulose bisphosphate carboxylase small chain (RBCS)}

$R B C S 2$ is routinely used as a constitutive promoter for nuclear transgene expression in tandem with HSP70A [79-81]. RBCS expression was upregulated in both phototrophic and mixotrophic cultures grown under red light illumination (RA, RX; Fig. 2A). Fig. 7 presents $R B C S$ expression data for an additional time point in the late stationary phase of the culture. In agreement with the results of Fig. 2A, RBCS expression is upregulated significantly for cultures grown under red light (RA, RX; Fig. 7A). Interestingly, there is a large increase in RBCS expression during the stationary phase $(136 \mathrm{~h})$ for both mixotrophic and phototrophic cultures grown in red light. These results suggest that red light illumination would be preferable for recombinant protein synthesis when RBSC2 is used as a constitutive promoter.

\subsubsection{Heat shock protein 70A (HSP70A)}

$H S P 70 A$ encodes a heat-shock protein, transcriptionally controlled through elevated temperatures and light [79]. It has been used as a transcriptional activator of other promoters, most commonly RBCS2 and recently $R B C S 2$ with $R B C S 2$ introns interspersed in the recombinant gene coding sequence [52]. In photoautotrophic cultures, a notable increase in expression was only observed during the stationary phase (136 h) of cultures grown in red light (Fig. 7B). HSP70A expression levels were higher in the stationary phase of mixotrophic cultures across all wavelengths with a notable 6 -fold increase $(p<0.05)$ observed in cultures grown under blue light illumination (Fig. 7B).

\subsubsection{Adenosine triphosphate synthase subunit alpha (atpA)}

Assembly of chloroplastic ATP-synthase requires subunits encoded in both the nuclear and the chloroplast genomes [82]. The gene atpA is native to the plastome and encodes sub-unit alpha of the $\mathrm{CF}_{1}$ complex [83]. Its 5'UTR and promoter coding regions have been used to drive both nuclear [84-86] and plastid [87,88] gene expression. Cultures grown under white light illumination had the lowest levels of atpA expression across all conditions examined (Fig. 7). Interestingly, trophic strategy had a significant impact on atpA expression only in cultures grown under red light where a 5.5 fold upregulation was observed in mixotrophic cultures during the mid-exponential phase ( $16 \mathrm{~h}$ and $48 \mathrm{~h}$ ). These results confirm that culture time, trophic strategy and wavelength selection can all have a significant impact on the transcriptional activity of promoters and should be considered as critical process parameters during process development and optimisation. 

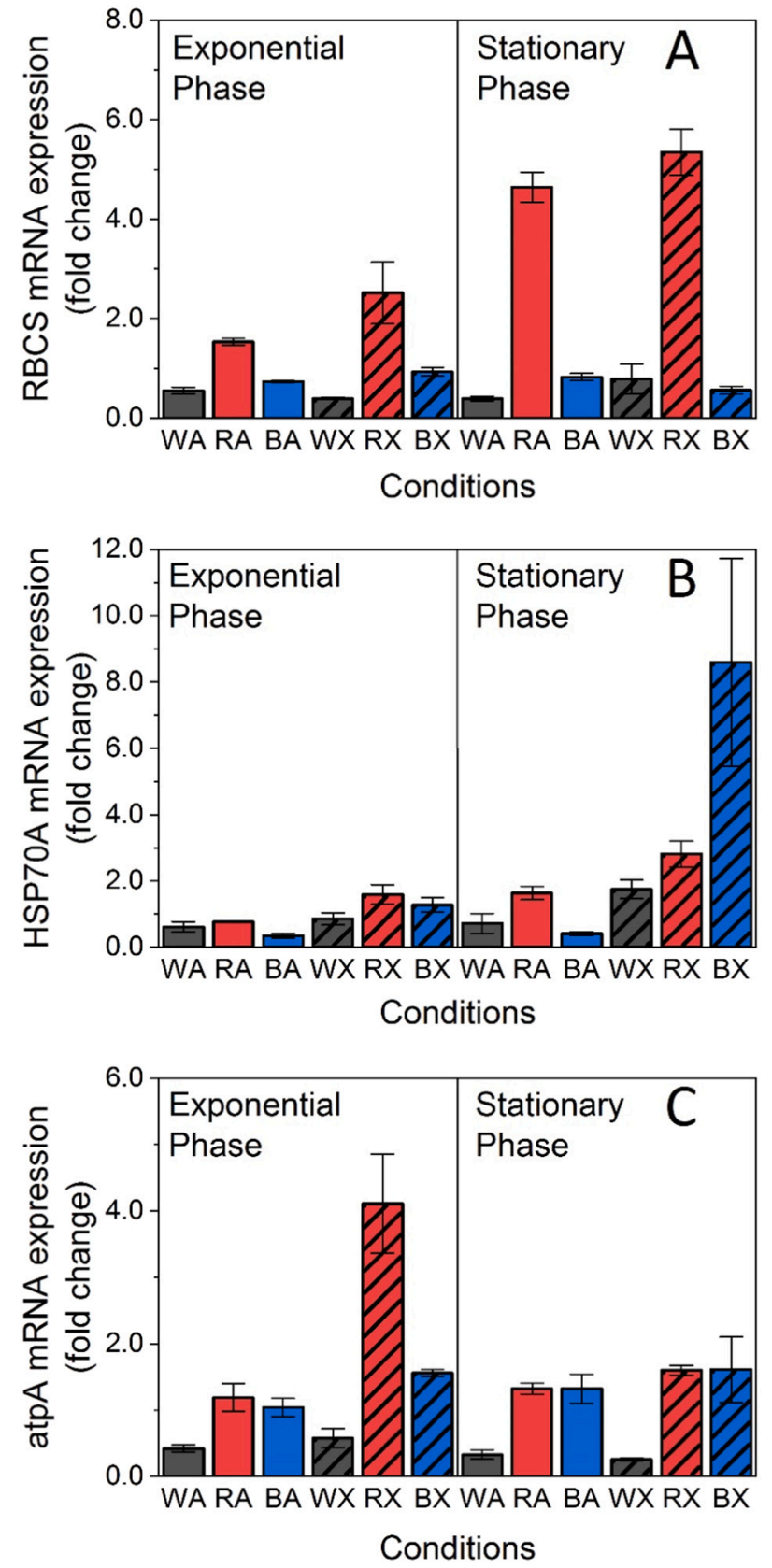

Fig. 7. Average gene expression of (A) ribulose bisphosphate carboxylase small subunit - RBCS, (B) heat-shock protein 70A - HSP70A and (C) ATP synthase subunit alpha $(\operatorname{atp} A)$ respectively, from $16 \mathrm{~h}-48 \mathrm{~h}$ (Exponential Phase, $\mathrm{n}=4$, error bars represent SEM) and $136 \mathrm{~h}$ (Stationary Phase) $(n=2$, error bars represent min and max expression). W: white light, R: red light 640-670 nm, B: blue light 440-480 nm, A: phototrophic growth in M8a, X: mixotrophic growth in M8a.Ac. (For interpretation of the references to colour in this figure legend, the reader is referred to the web version of this article.)

\section{Conclusions}

The expression profiles of a total of 16 genes (Table 1 ) were monitored across $C$. reinhardtii cultures grown under two different trophic strategies and three different wavelengths. This enabled the identification of novel reference genes ( $p s a D$ and $A C X 1$ ) with significantly improved expression stability compared to commonly used reference genes, for the conditions explored herein. The analysis of the expression profiles of representative genes from key metabolic pathways revealed that trophic strategy and wavelength selection have an impact on some
(RBCS - photosynthesis, MAS1 - glyoxylate cycle, KAS2 - fatty acid synthesis) but not all (MAS1 - TCA cycle) aspects of central carbon metabolism in algae. Specifically, wavelength selection was found to have an impact on photosynthetic efficiency and (as indicated by cell size distribution data) the cell cycle. Moreover, based on the set of gene expression profiles examined in the present study, assimilated acetate was found to be primarily channelled towards fatty acid synthesis, particularly in cultures grown under red light illumination. Finally, the expression profiles of three commonly used promoters for the expression of recombinant proteins were evaluated across all experimental conditions and revealed that culture time, trophic strategy and wavelength selection should be considered as critical process parameters during process development and optimisation. The evaluation of a panel of candidate reference genes under the diverse conditions examined herein highlighted the importance of establishing a priori a systematic workflow that facilitates reliable comparisons across culture conditions and will hopefully inform the experimental design of future studies.

\section{CRediT authorship contribution statement}

Victor Sanchez Tarre: Conceptualization, Methodology, Validation, Formal analysis, Data Curation, Investigation, Writing - Original Draft, Visualization.

Alexandros Kiparissides: Conceptualization, Formal analysis, Data Curation, Project administration, Funding acquisition, Resources, Writing - Review \& Editing, Supervision.

\section{Declaration of competing interest}

The authors declare that they have no conflict of interests.

\section{Acknowledgements}

Funding from the UK Engineering and Physical Sciences Research Council (EPSRC) through the EPSRC Centre for Doctoral Training in Emergent Macromolecular Therapies (EMT) grant EP/L015218/1 is gratefully acknowledged.

\section{Appendix A. Supplementary data}

Supplementary data to this article can be found online at https://doi. org/10.1016/j.algal.2021.102186.

\section{References}

[1] A.R. Grossman, M. Croft, V.N. Gladyshev, S.S. Merchant, M.C. Posewitz, S. Prochnik, M.H. Spalding, Novel metabolism in Chlamydomonas through the lens of genomics, Curr. Opin. Plant Biol. 10 (2007) 190-198, https://doi.org/10.1016/ j.pbi.2007.01.012.

[2] A. Jungandreas, B.S. Costa, T. Jakob, M. Von Bergen, S. Baumann, C. Wilhelm, The acclimation of Phaeodactylum tricornutum to blue and red light does not influence the photosynthetic light reaction but strongly disturbs the carbon allocation pattern, PLoS One. 9 (2014), https://doi.org/10.1371/journal.pone.0099727.

[3] O.A. Sineshchekov, K.-H. Jung, J.L. Spudich, Two rhodopsins mediate phototaxis to low- and high-intensity light in Chlamydomonas reinhardtii, Proc. Natl. Acad. Sci. 99 (2002) 8689-8694, https://doi.org/10.1073/pnas.122243399.

[4] K. Huang, C.F. Beck, Phototropin is the blue-light receptor that controls multiple steps in the sexual life cycle of the green alga Chlamydomonas reinhardtii, Proc. Natl. Acad. Sci. 100 (2003) 6269-6274, https://doi.org/10.1073/ pnas.0931459100.

[5] N. Müller, S. Wenzel, Y. Zou, S. Künzel, S. Sasso, D. Weiß, K. Prager, A. Grossman, T. Kottke, M. Mittag, A plant Cryptochrome controls key features of the Chlamydomonas circadian clock and its life cycle, Plant Physiol. 174 (2017) 185-201, https://doi.org/10.1104/pp.17.00349.

[6] M.D. Ooms, C.T. Dinh, E.H. Sargent, D. Sinton, Photon management for augmented photosynthesis, Nat. Commun. 7 (2016) 12699, https://doi.org/10.1038/ ncomms12699.

[7] J. Steinbrenner, H. Linden, Light induction of carotenoid biosynthesis genes in the green alga Haematococcus pluvialis: regulation by photosynthetic redox control, Plant Mol. Biol. 52 (2003) 343-356, https://doi.org/10.1023/A:1023948929665.

[8] P.P. Lamers, M. Janssen, R.C.H. De Vos, R.J. Bino, R.H. Wijffels, Exploring and exploiting carotenoid accumulation in Dunaliella salina for cell-factory applications, 
Trends Biotechnol. 26 (2008) 631-638, https://doi.org/10.1016/j. tibtech.2008.07.002.

[9] A.P. Carvalho, S.O. Silva, J.M. Baptista, F.X. Malcata, Light requirements in microalgal photobioreactors: an overview of biophotonic aspects, Appl. Microbiol Biotechnol. 89 (2011) 1275-1288, https://doi.org/10.1007/s00253-010-3047-8.

[10] I. Wagner, C. Steinweg, C. Posten, Mono- and dichromatic LED illumination leads to enhanced growth and energy conversion for high-efficiency cultivation of microalgae for application in space, Biotechnol. J. 11 (2016) 1060-1071, https:// doi.org/10.1002/biot.201500357.

[11] T. De Mooij, G. De Vries, C. Latsos, R.H. Wijffels, M. Janssen, Impact of light color on photobioreactor productivity, Algal Res. 15 (2016) 32-42, https://doi.org/ 10.1016/j.algal.2016.01.015.

[12] P.M. Pattison, J.Y. Tsao, G.C. Brainard, B. Bugbee, LEDs for photons, physiology and food, Nature. 563 (2018) 493-500, https://doi.org/10.1038/s41586-0180706-x.

[13] S. Abu-Ghosh, D. Fixler, Z. Dubinsky, D. Iluz, Flashing light in microalgae biotechnology, Bioresour. Technol. 203 (2016) 357-363, https://doi.org/10.1016/ j.biortech.2015.12.057.

[14] Z. Amini Khoeyi, J. Seyfabadi, Z. Ramezanpour, Effect of light intensity and photoperiod on biomass and fatty acid composition of the microalgae, Chlorella vulgaris, Aquac. Int. 20 (2012) 41-49, https://doi.org/10.1007/s10499-011-94401.

[15] S.K. Wang, A.R. Stiles, C. Guo, C.Z. Liu, Microalgae cultivation in photobioreactors: an overview of light characteristics, Eng. Life Sci. 14 (2014) 550-559, https://doi org/10.1002/elsc.201300170.

[16] P.S.C. Schulze, L.A. Barreira, H.G.C. Pereira, J.A. Perales, J.C.S. Varela, Ligh emitting diodes (LEDs) applied to microalgal production, Trends Biotechnol. 32 (2014) 422-430, https://doi.org/10.1016/j.tibtech.2014.06.001.

[17] R. Guo, M.A. Lee, J.S. Ki, Normalization genes for mRNA expression in the marine diatom Ditylum brightwellii following exposure to thermal and toxic chemical stresses, J. Appl. Phycol. 25 (2013) 1101-1109, https://doi.org/10.1007/s10811 012-9908-Z.

[18] N.N. Rosic, M. Pernice, M. Rodriguez-Lanetty, O. Hoegh-Guldberg, Validation of housekeeping genes for gene expression studies in Symbiodinium exposed to thermal and light stress, Mar. Biotechnol. 13 (2011) 355-365, https://doi.org/ 10.1007/s10126-010-9308-9.

[19] C. Liu, G. Wu, X. Huang, S. Liu, B. Cong, Validation of housekeeping genes for gen expression studies in an ice alga Chlamydomonas during freezing acclimation, Extremophiles. 16 (2012) 419-425, https://doi.org/10.1007/s00792-012-0441-4.

[20] R.T. Smith, D.J. Gilmour, The influence of exogenous organic carbon assimilation and photoperiod on the carbon and lipid metabolism of Chlamydomonas reinhardtii, Algal Res. 31 (2018) 122-137, https://doi.org/10.1016/j. algal.2018.01.020.

[21] S. Schmollinger, T. Mühlhaus, N.R. Boyle, I.K. Blaby, D. Casero, T. Mettler, J. L. Moseley, J. Kropat, F. Sommer, D. Strenkert, D. Hemme, M. Pellegrini, A. R. Grossman, M. Stitt, M. Schroda, S.S. Merchant, Nitrogen-sparing mechanisms in Chlamydomonas affect the transcriptome, the proteome, and photosynthetic metabolism, Plant Cell 26 (2014) 1410-1435, https://doi.org/10.1105/ tpc.113.122523.

[22] A.M.J. Kliphuis, L. de Winter, C. Vejrazka, D.E. Martens, M. Janssen, R.H. Wijffels, Photosynthetic efficiency of Chlorella sorokiniana in a turbulently mixed short light-path photobioreactor, Biotechnol. Prog. 26 (2010) 687-696, https://doi.org/ 10.1002/btpr.379.

[23] R. Sager, S. Granick, Nutritional studies with Chlamydomonas reinhardtii, Ann. N. Y. Acad. Sci. 56 (1953) 831-838, https://doi.org/10.1111/j.1749-6632.1953. tb30261.x.

[24] W.P. Inskeep, P.R. Bloom, Extinction coefficients of chlorophyll-a and chlorophyllb in n,ndimethylformamide and 80-percent acetone, Plant Physiol. 77 (1985) 483-485.

[25] M. Rosner, K. Hofer, M. Kubista, M. Hengstschläger, Cell size regulation by the human TSC tumor suppressor proteins depends on PI3K and FKBP38, Oncogene. 22 (2003) 4786-4798, https://doi.org/10.1038/sj.onc.1206776.

[26] D.M. Goodstein, S. Shu, R. Howson, R. Neupane, R.D. Hayes, J. Fazo, T. Mitros, W. Dirks, U. Hellsten, N. Putnam, D.S. Rokhsar, Phytozome: a comparative platform for green plant genomics, Nucleic Acids Res. 40 (2011) D1178-D1186, https://doi.org/10.1093/nar/gkr944.

[27] A. Untergasser, I. Cutcutache, T. Koressaar, J. Ye, B.C. Faircloth, M. Remm, S. G. Rozen, Primer3-new capabilities and interfaces, Nucleic Acids Res. 40 (2012), https://doi.org/10.1093/nar/gks596.

[28] P. Stothard, The sequence manipulation suite: JavaScript programs for analyzing and formatting protein and DNA sequences, Biotechniques. 28 (2000) 1102-1104.

[29] J. Vandesompele, K. De Preter, F. Pattyn, B. Poppe, N. Van Roy, A. De Paepe, F. Speleman, Accurate normalization of real-time quantitative RT-PCR data by geometric averaging of multiple internal control genes, Genome Biol. 3 (2002), https://doi.org/10.1186/gb-2002-3-7-research0034 research0034.1.

[30] J. Hellemans, G. Mortier, A. De Paepe, F. Speleman, J. Vandesompele, qBase relative quantification framework and software for management and automated analysis of real-time quantitative PCR data, Genome Biol. 8 (2007) R19, https:// doi.org/10.1186/gb-2007-8-2-r19.

[31] F. Perozeni, S. Cazzaniga, T. Baier, F. Zanoni, G. Zoccatelli, K.J. Lauersen, L. Wobbe, M. Ballottari, Turning a green alga red: engineering astaxanthin biosynthesis by intragenic pseudogene revival in Chlamydomonas reinhardtii, Plant Biotechnol. J. 18 (2020) 2053-2067, https://doi.org/10.1111/pbi.13364.

[32] S.E. Carrera Pacheco, B. Hankamer, M. Oey, Optimising light conditions increase recombinant protein production in Chlamydomonas reinhardtii chloroplasts, Algal Res. 32 (2018) 329-340, https://doi.org/10.1016/j.algal.2018.04.011.
[33] J.P. Rathod, C. Vira, A.M. Lali, G. Prakash, Metabolic engineering of Chlamydomonas reinhardtii for enhanced $\beta$-carotene and lutein production, Appl. Biochem. Biotechnol. 190 (2020) 1457-1469, https://doi.org/10.1007/s12010019-03194-9.

[34] S. Sivakaminathan, B. Hankamer, J. Wolf, J. Yarnold, High-throughput optimisation of light-driven microalgae biotechnologies, Sci. Rep. 8 (2018) 11687, https://doi.org/10.1038/s41598-018-29954-x.

[35] G.L. Shipley, An introduction to real-time PCR, in: D.T. M (Ed.), Real-Time PCR, 1st ed., Taylor \& Francis, 2006, pp. 1-37.

[36] M. Siaut, M. Heijde, M. Mangogna, A. Montsant, S. Coesel, A. Allen, A. Manfredonia, A. Falciatore, C. Bowler, Molecular toolbox for studying diatom biology in Phaeodactylum tricornutum, Gene. 406 (2007) 23-35, https://doi.org/ 10.1016/j.gene.2007.05.022.

[37] B. Beel, K. Prager, M. Spexard, S. Sasso, D. Weiss, N. Muller, M. Heinnickel, D. Dewez, D. Ikoma, A.R. Grossman, T. Kottke, M. Mittag, A flavin binding cryptochrome photoreceptor responds to both blue and red light in Chlamydomonas reinhardtii, Plant Cell. 24 (2012) 2992-3008, https://doi.org/10.1105/ tpc.112.098947.

[38] A. Kianianmomeni, A. Hallmann, Validation of reference genes for quantitative gene expression studies in Volvox carteri using real-time RT-PCR, Mol. Biol. Rep. 40 (2013) 6691-6699, https://doi.org/10.1007/s11033-013-2784-z.

[39] M.G. Adelfi, M. Borra, R. Sanges, M. Montresor, A. Fontana, M.I. Ferrante, Selection and validation of reference genes for qPCR analysis in the pennate diatoms Pseudo-nitzschia multistriata and P. arenysensis, J. Exp. Mar. Bio. Ecol. 451 (2014) 74-81, https://doi.org/10.1016/j.jembe.2013.11.003.

[40] J. Li, H. Huang, T. Shan, S. Pang, Selection of reference genes for real-time RT-PCR normalization in brown alga Undaria pinnatifida, J. Appl. Phycol. 31 (2018) 787-793, https://doi.org/10.1007/s10811-018-1576-1.

[41] K.J. Livak, T.D. Schmittgen, Analysis of relative gene expression data using realtime quantitative PCR and the $2(-\Delta \Delta \mathrm{C}(\mathrm{T}))$ method, Methods. 25 (2001) 402-408, https://doi.org/10.1006/meth.2001.1262.

[42] M.W. Pfaffl, A new mathematical model for relative quantification in real-time RTPCR, Nucleic Acids Res. 29 (2001), e45, https://doi.org/10.1093/nar/29.9.e45.

[43] S. Derveaux, J. Vandesompele, J. Hellemans, How to do successful gene expression analysis using real-time PCR, Methods. 50 (2010) 227-230, https://doi.org/ 10.1016/j.ymeth.2009.11.001.

[44] A. Cortleven, T. Remans, W.G. Brenner, R. Valcke, Selection of plastid- and nuclear-encoded reference genes to study the effect of altered endogenous cytokinin content on photosynthesis genes in Nicotiana tabacum, Photosynth. Res. 102 (2009) 1-9, https://doi.org/10.1007/s11120-009-9470-y.

[45] A. Cankorur-Cetinkaya, E. Dereli, S. Eraslan, E. Karabekmez, D. Dikicioglu, B. Kirdar, A novel strategy for selection and validation of reference genes in dynamic multidimensional experimental design in yeast, PLoS One 7 (2012) 1-14, https://doi.org/10.1371/journal.pone.0038351.

[46] J. Ke, T.N. Wen, B.J. Nikolau, E.S. Wurtele, Coordinate regulation of the nuclear and plastidic genes coding for the subunits of the heteromeric acetyl-coenzyme A carboxylase, Plant Physiol. 122 (2000) 1057-1071, https://doi.org/10.1104/ pp.122.4.1057.

[47] U. Goodenough, I. Blaby, D. Casero, S.D. Gallaher, C. Goodson, S. Johnson, J. H. Lee, S.S. Merchant, M. Pellegrini, R. Roth, J. Rusch, M. Singh, J.G. Umen, T. L. Weiss, T. Wulan, The path to triacylglyceride obesity in the sta6 strain of Chlamydomonas reinhardtii, Eukaryot. Cell 13 (2014) 591-613, https://doi.org/ 10.1128/EC.00013-14.

[48] R. Ramanan, B.H. Kim, D.H. Cho, S.R. Ko, H.M. Oh, H.S. Kim, Lipid droplet synthesis is limited by acetate availability in starchless mutant of Chlamydomonas reinhardtii, FEBS Lett. 587 (2013) 370-377, https://doi.org/10.1016/j. febslet.2012.12.020.

[49] A. Ihnatowicz, P. Pesaresi, C. Varotto, E. Richly, A. Schneider, P. Jahns, F. Salamini, D. Leister, Mutants for photosystem I subunit D of Arabidopsis thaliana: effects on photosynthesis, photosystem I stability and expression of nuclear genes for chloroplast functions, Plant J. 37 (2004) 839-852, https://doi. $\operatorname{org} / 10.1111 / \mathrm{j} .1365-313 X .2004 .02011 . x$.

[50] P.R. Chitnis, P.A. Reilly, N. Nelson, Insertional inactivation of the gene encoding subunit II of photosystem I from the cyanobacterium Synechocystis sp. PCC 6803, J. Biol. Chem. 264 (1989) 18381-18385.

[51] N. Fischer, J.D. Rochaix, The flanking regions of PsaD drive efficient gene expression in the nucleus of the green alga Chlamydomonas reinhardtii, Mol. Gen. Genomics. 265 (2001) 888-894, https://do1.org/10.1007/s004380100485.

[52] T. Baier, J. Wichmann, O. Kruse, K.J. Lauersen, Intron-containing algal transgenes mediate efficient recombinant gene expression in the green microalga Chlamydomonas reinhardtii, Nucleic Acids Res. 46 (2018) 6909-6919, https://doi. org/10.1093/nar/gky532.

[53] Y. Choquet, F.-A. Wollman, The CES process, Chlamydomonas Sourceb. Organellar Metab. Process. (2009) 1027-1064.

[54] I. Khrebtukova, R.J. Spreitzer, Elimination of the Chlamydomonas gene family that encodes the small subunit of ribulose-1,5-bisphosphate carboxylase/oxygenase, Proc. Natl. Acad. Sci. 93 (1996) 13689-13693, https://doi.org/10.1073/ pnas.93.24.13689.

[55] M. Goldschmidt-Clermont, M. Rahire, Sequence, evolution and differentia expression of the two genes encoding variant small subunits of ribulose bisphosphate carboxylase/oxygenase in Chlamydomonas reinhardtii, J. Mol. Biol. 191 (1986) 421-432, https://doi.org/10.1016/0022-2836(86)90137-3.

[56] M. Goldschmidt-Clermont, The two genes for the small subunit of RuBP carboxylase/oxygenase are closely linked in Chlamydomonas reinhardtii, Plant Mol. Biol. 6 (1986) 13-21, https://doi.org/10.1007/BF00021302. 
[57] H. Oldenhof, V. Zachleder, H. Van Den Ende, Blue- and red-light regulation of the cell cycle in chlamydomonas reinhardtii (Chlorophyta), Eur. J. Phycol. 41 (2006) 313-320, https://doi.org/10.1080/09670260600699920.

[58] H. Oldenhof, V. Zachleder, H. Van Den Ende, Blue light delays commitment to cell division in Chlamydomonas reinhardtii, Plant Biol. 6 (2004) 689-695, https://doi. org/10.1055/s-2004-821341.

[59] Z. Perrine, S. Negi, R.T. Sayre, Optimization of photosynthetic light energy utilization by microalgae, Algal Res. 1 (2012) 134-142, https://doi.org/10.1016/j. algal.2012.07.002

[60] X. Johnson, J. Alric, Interaction between starch breakdown, acetate assimilation, and photosynthetic cyclic electron flow in Chlamydomonas reinhardtii, J. Biol. Chem. 287 (2012) 26445-26452, https://doi.org/10.1074/jbc.M112.370205.

[61] H. Singh, M.R. Shukla, K.V.R. Chary, B.J. Rao, Acetate and bicarbonate assimilation and metabolite formation in Chlamydomonas reinhardtii: A 13C-NMR study, PLoS One. 9 (2014), https://doi.org/10.1371/journal.pone.0106457.

[62] K.J. Lauersen, R. Willamme, N. Coosemans, M. Joris, O. Kruse, C. Remacle, Peroxisomal microbodies are at the crossroads of acetate assimilation in the green microalga Chlamydomonas reinhardtii, Algal Res. 16 (2016) 266-274, https://doi. org/10.1016/j.algal.2016.03.026.

[63] M. Terashima, M. Specht, B. Naumann, M. Hippler, Characterizing the Anaerobic response of Chlamydomonas reinhardtii by quantitative proteomics, Mol. Cell. Proteomics. 9 (2010) 1514-1532, https://doi.org/10.1074/mcp.M900421MCP200.

[64] J.A. Raven, J. Beardall, Dark respiration and organic carbon loss, in: M. A. Borowitzka, J.A. Raven (Eds.), Physiol. Microalgae, 1st ed., Springer, 2016, pp. 129-142.

[65] B.E. Rubin, K.M. Wetmore, M.N. Price, S. Diamond, R.K. Shultzaberger, L.C. Lowe, G. Curtin, A.P. Arkin, A. Deutschbauer, S.S. Golden, The essential gene set of a photosynthetic organism, Proc. Natl. Acad. Sci. 112 (2015) E6634-E6643, https:// doi.org/10.1073/pnas.1519220112.

[66] A.H. Millar, J. Whelan, K.L. Soole, D.A. Day, Organization and regulation of mitochondrial respiration in plants, Annu. Rev. Plant Biol. 62 (2011) 79-104, https://doi.org/10.1146/annurev-arplant-042110-103857.

[67] B.A. Silverberg, An ultrastructural and cytochemical characterization of microbodies in the green algae, Protoplasma. 83 (1975) 269-295, https://doi.org/ 10.1007/BF01282559.

[68] S.P. Chapman, C.M. Paget, G.N. Johnson, J.-M. Schwartz, Flux balance analysis reveals acetate metabolism modulates cyclic electron flow and alternative glycolytic pathways in Chlamydomonas reinhardtii, Front. Plant Sci. 6 (2015) 1-14, https://doi.org/10.3389/fpls.2015.00474.

[69] Y. Hayashi, N. Sato, A. Shinozaki, M. Watanabe, Increase in peroxisome number and the gene expression of putative glyoxysomal enzymes in Chlamydomonas cells supplemented with acetate, J. Plant Res. 128 (2014) 177-185, https://doi.org/ 10.1007/s10265-014-0681-8.

[70] H. Wang, B. Gau, W.O. Slade, M. Juergens, P. Li, L.M. Hicks, The global phosphoproteome of chlamydomonas reinhardtii reveals complex organellar phosphorylation in the flagella and thylakoid membrane, Mol. Cell. Proteomics 13 (2014) 2337-2353, https://doi.org/10.1074/mcp.M114.038281.

[71] W.R. Riekhof, C. Benning, Glycerolipid biosynthesis, in: D.B. Stern (Ed.), Chlamydomonas Sourceb. Organellar Metab. Process., 2nd ed., Elsevier, 2009, pp. 41-69, https://doi.org/10.1016/0092-8674(90)90467-S.

[72] Y. Li-Beisson, F. Beisson, W. Riekhof, Metabolism of acyl-lipids in Chlamydomonas reinhardtii, Plant J. 82 (2015) 504-522, https://doi.org/10.1111/tpj.12787.

[73] M.P. Davey, I. Horst, G.H. Duong, E.V. Tomsett, A.C.P. Litvinenko, C.J. Howe, A. G. Smith, Triacylglyceride production and Autophagous responses in
Chlamydomonas reinhardtii depend on resource allocation and carbon source, Eukaryot. Cell 13 (2014) 392-400, https://doi.org/10.1128/EC.00178-13.

[74] S. Jeon, J.M. Lim, H.G. Lee, S.E. Shin, N.K. Kang, Y. Il Park, H.M. Oh, W.J. Jeong, B.R. Jeong, Y.K. Chang, Current status and perspectives of genome editing technology for microalgae, Biotechnol. Biofuels. 10 (2017) 1-18, https://doi.org/ 10.1186/s13068-017-0957-z.

[75] H.N. Taunt, L. Stoffels, S. Purton, Green Biologics: The Algal Chloroplast as A Platform for Making Biopharmaceuticals, 5979, 2018, https://doi.org/10.1080/ 21655979.2017.1377867.

[76] M. Nymark, A.K. Sharma, T. Sparstad, A.M. Bones, P. Winge, A CRISPR/Cas9 system adapted for gene editing in marine algae, Sci. Rep. 6 (2016) 6-11, https:// doi.org/10.1038/srep24951.

[77] S.P. Fletcher, M. Muto, S.P. Mayfield, Optimization of recombinant protein expression in the chloroplasts of green algae, in: R. León, A. Galván, E. Fernández (Eds.), Transgenic Microalgae as Green Cell Factories, Springer New York, New York, NY, 2007, pp. 90-98, https://doi.org/10.1007/978-0-387-75532-8_8.

[78] B.A. Rasala, S.P. Mayfield, Photosynthetic biomanufacturing in green algae; production of recombinant proteins for industrial, nutritional, and medical uses, Photosynth. Res. 123 (2015) 227-239, https://doi.org/10.1007/s11120-0149994-7.

[79] M. Schroda, D. Blöcker, C.F. Beck, The HSP70A promoter as a tool for the improved expression of transgenes in Chlamydomonas, Plant J. 21 (2000) 121-131, https:// doi.org/10.1046/j.1365-313X.2000.00652.x.

[80] B.A. Rasala, S.S. Chao, M. Pier, D.J. Barrera, S.P. Mayfield, Enhanced genetic tools for engineering multigene traits into green algae, PLoS One 9 (2014), e94028, https://doi.org/10.1371/journal.pone.0094028.

[81] T. Baier, D. Kros, R.C. Feiner, K.J. Lauersen, K.M. Müller, O. Kruse, Engineered fusion proteins for efficient protein secretion and purification of a human growth factor from the green microalga Chlamydomonas reinhardtii, ACS Synth. Biol. 7 (2018) 2547-2557, https://doi.org/10.1021/acssynbio.8b00226.

[82] G. Finazzi, D. Drapier, F. Rappaport, The CFOF1 ATP synthase complex of photosynthesis, in: D.B. Stern (Ed.), Chlamydomonas Sourceb. Organellar Metab. Process., 2nd ed., Elsevier, 2009, pp. 639-670, https://doi.org/10.1016/00928674(90)90467-S.

[83] H. Levy, K.L. Kindle, D.B. Stern, A nuclear mutation that affects the 3[prime] processing of several mRNAs in chlamydomonas chloroplasts, Plant Cell. 9 (1997) 825-836, https://doi.org/10.1105/tpc.9.5.825.

[84] K. Ishikura, Y. Takaoka, K. Kato, M. Sekine, K. Yoshida, A. Shinmyo, Expression of a foreign gene in Chlamydomonas reinhardtii chloroplast, J. Biosci. Bioeng. 87 (1999) 307-314, https://doi.org/10.1016/S1389-1723(99)80037-1.

[85] S. Kasai, S. Yoshimura, K. Ishikura, Y. Takaoka, K. Kobayashi, K. Kato, A. Shinmyo, Effect of coding regions on chloroplast gene expression in Chlamydomonas reinhardtii, J. Biosci. Bioeng. 95 (2003) 276-282, https://doi.org/10.1016/s13891723(03)80029-4.

[86] L. Michelet, L. Lefebvre-Legendre, S.E. Burr, J.-D. Rochaix, M. GoldschmidtClermont, Enhanced chloroplast transgene expression in a nuclear mutant of Chlamydomonas, Plant Biotechnol. J. 9 (2011) 565-574, https://doi.org/10.1111/ j.1467-7652.2010.00564.x.

[87] I. Bertalan, M.C. Munder, C. Weiß, J. Kopf, D. Fischer, U. Johanningmeier, A rapid, modular and marker-free chloroplast expression system for the green alga Chlamydomonas reinhardtii, J. Biotechnol. 195 (2015) 60-66, https://doi.org/ 10.1016/j.jbiotec.2014.12.017.

[88] S. Braun-Galleani, F. Baganz, S. Purton, Improving recombinant protein production in the Chlamydomonas reinhardtii chloroplast using vivid Verde fluorescent protein as a reporter, Biotechnol. J. 10 (2015) 1289-1297, https://doi.org/ 10.1002/biot.201400566. 